\title{
Quasi-one-dimensional charge density wave in electromagnetic field arbitrarily oriented to conducting chains: generalized Fröhlich relations
}

\author{
Alexander S. Rozhavsky ${ }^{(1,2, a)}$, Yurij V. Pershin ${ }^{(1,2)}$ and Igor A. Romanovsky ${ }^{(1,3)}$ \\ ${ }^{1}$ B.I. Verkin Institute for Low Temperature Physics and Engineering, \\ 47 Lenin Avenue 310164 Kharkov, Ukraine \\ (permanent address) \\ ${ }^{2}$ Grenoble High Magnetic Field Laboratory, \\ Max-Planck-Institut für Festkörperforschung and CNRS, \\ BP 166, F-38042, Grenoble, Cedex 9, France \\ ${ }^{3}$ Physical Department, Kharkov State University, \\ 4 Svobody Square 310077 Kharkov, Ukraine
}

\begin{abstract}
We derive equations for the collective CDW-current transverse conducting chains in a quasi-one-dimensional CDW-conductor. Generalized Fröhlich relations between the transverse currents and phase gradients are due to the polarization corrections to the $1+1$ chiral anomaly Lagrangean. The CDW Hall constant $R_{C D W}$ is calculated, $R_{C D W} \sim$ $T_{C}^{2} / I_{C D W}$, where $T_{C}$ is the critical temperature of the Peierls transition, and $I_{C D W}$ is the nonlinear CDW current in the direction parallel to the conducting chains.
\end{abstract}

PACS numbers: $71.45 \mathrm{Lr}, 72.15 \mathrm{Nj}, 11.30 \mathrm{Rd}$

a) Corresponding author. E-mail: rozhavsky@ilt.kharkov.ua 
The Quasi-One-Dimensional (Q1D) Charge Density Wave (CDW) conductors such as $\mathrm{NbSe}_{3}, \mathrm{TaS}_{3}, \mathrm{~K}_{0,3} \mathrm{MoO}_{3}$ etc. (see for instance, Ref.[1]) are characterized by a strongly anisotropic quasiparticle spectra. As a result, their unusual transport properties are mostly pronounced in the direction parallel to the conducting chains. The theoretical studies of the CDW-electrodynamics were mainly focused on the one-dimensional aspect. The response of a 1D CDW is described by the well-known Fröhlich relations [1]:

$$
\begin{gathered}
j_{x}=-\frac{e}{\pi} \frac{\partial \varphi}{\partial t} n_{f} \\
\rho=\frac{e}{\pi} \frac{\partial \varphi}{\partial x} n_{f}
\end{gathered}
$$

where $j_{x}$ is the collective current density along the direction of the chains, $\rho$ is the charge density fluctuation, $n_{f}$ is the density of chains and $\varphi$ is the CDW-variable, the phase of the Peierls-Fröhlich order parameter $\Delta \exp (i \varphi)$. The amplitude $\Delta$ is equal to the gap width in the single-particle spectrum.

Experimental investigations of the transverse CDW transport, and in particular of the Hall effect [2, 3], have demonstrated that the 3D-effects are also strongly affected by the nonlinear CDW transport current $I_{C D W}$ directed along the chains. It was found in Ref. [3] that the Hall constant $R_{C D W}$ is proportional to $I_{C D W}^{-1}$ and sharply decreases in electric fields above the threshold $E_{T}$. This fact is not yet explained within the microscopic theory. To describe properly the 3D electrodynamics of CDW, we need the generalized Fröhlich equations for all the three components of the collective current $\vec{I}_{C D W}$. This problem is solved in the present paper, and the dependence of $R_{C D W}$ on $I_{C D W}$ is obtained.

The description of a Q1D CDW is based on the electron-lattice Hamiltonian:

$$
\begin{gathered}
\hat{H}=-\frac{\hbar^{2}}{2 M} \sum_{\vec{n}_{\nu}} \frac{\partial^{2}}{\partial u_{\vec{n}_{\nu}}^{2}}+\sum_{\vec{n}_{\nu}} K_{\nu}\left(u_{\vec{n}_{\nu}}-u_{\vec{n}_{\nu}+\overrightarrow{1}}\right)^{2}+ \\
\sum_{\vec{n}_{\nu}}\left(t_{\nu}+t_{1 \nu}\left(u_{\vec{n}_{\nu}}-u_{\vec{n}_{\nu}+\overrightarrow{1}}\right)\right)\left(a_{\vec{n}_{\nu}, s}^{+} a_{\vec{n}_{\nu}+\overrightarrow{1}, s}+\text { h.c. }\right)
\end{gathered}
$$

where $u_{\vec{n}}$ is the lattice displacement, $\vec{n}$ numerates lattice sites, $\nu=x, y, z, M$ is the ion mass, $K_{\nu}$ are the lattice elasticity constants, $t_{\nu}$ is the electron hopping integral, $t_{1}$ is the electron-lattice coupling, $a_{\vec{n} s}^{+}$and $a_{\vec{n} s}$ are the creation and annihilation operators of an electron with spin $s$ at the site $\vec{n}$.

The Q1D approximation is formulated as follows:

$$
\begin{gathered}
u_{n_{x}}=u_{0} \cos \left(2 k_{F} n_{x} b_{x}+\varphi\right) \\
a_{\vec{n} s}=\Psi_{R s} \exp \left(i k_{F} n_{x} b_{x}\right)+\Psi_{L s} \exp \left(-i k_{F} n_{x} b_{x}\right) \\
u_{\vec{n}_{y, z}}-u_{\vec{n}+\overrightarrow{1}_{y, z}} \simeq \frac{\partial u_{\vec{n}}}{\partial y(z)} b_{y, z} \\
t_{1 y, z}=0
\end{gathered}
$$


For our purposes, it is convenient to formulate the theory in the Lagrange formalism.

After the standard redesignations (see e.g. [4]):

$$
b_{x} t_{0}=\hbar V_{F}, \quad 2 t_{1 x} u_{0} / b_{x}=\Delta, \quad K_{x} b_{x} \sin ^{2}\left(b_{x} k_{F}\right) / 16 t_{1 x}^{2}=\frac{1}{g^{2}},
$$

the Peierls-Fröhlich Lagrangean takes the form $(\hbar=c=1)$ :

$$
\begin{aligned}
\mathcal{L}= & \varphi\left(\frac{1}{\alpha^{2} V_{F}} \partial_{t}^{2}-\frac{U_{y}^{2}}{V_{F}} \partial_{y}^{2}-\frac{U_{z}^{2}}{V_{F}} \partial_{z}^{2}\right) \varphi-\frac{\Delta^{2}}{2 g^{2}}+\bar{\psi}\left\{i \sigma _ { 2 } \left(\partial_{t}+i e \Phi+i t_{y} \operatorname{ch}\left(b_{y}\left(\partial_{y}-i e A_{y}\right)\right)+\right.\right. \\
& \left.\left.i t_{z} \operatorname{ch}\left(b_{z}\left(\partial_{z}-i e A_{z}\right)\right)\right)-\sigma_{1} V_{F}\left(\partial_{x}-i e A_{x}\right)-\Delta \exp \left(-i \sigma_{3} \varphi\right)\right\} \psi=\mathcal{L}^{p h}+\bar{\psi} L \psi
\end{aligned}
$$

where the electromagnetic field is introduced in a gauge-invariant form. Here $\alpha^{2}=$ $\frac{\Delta}{V_{F}^{2} M}<<1$ is the adiabatic parameter of the Peierls-Fröhlich theory [⿴囗口, $U_{y, z}$ are the transverse phonon velocities, $\Phi$ and $A_{\nu}$ are the scalar and vector potentials, $\psi^{+}=\left(\psi_{R}^{+}, \psi_{L}^{+}\right)$, $\bar{\psi}=\psi^{+} \sigma_{2}$ and $\sigma_{\nu}$ - are the Pauli matrices. Note that the approximation (3) which leads to Eq. (4) is well defined only when $t_{y, z}<<\Delta$.

The CDW currents are:

$$
\begin{aligned}
j_{\nu} & =c \frac{\delta F}{\delta A_{\nu}} \\
\rho & =-\frac{\delta F}{\delta \Phi}
\end{aligned}
$$

where $F$ is the free energy:

$$
\begin{aligned}
& F=-T \ln \int D \Delta D \varphi D \bar{\psi} D \psi \exp \left(\int_{0}^{\frac{1}{T}} d \tau \int d \vec{r} \mathcal{L}_{E}\right)= \\
& -T \ln \int D \Delta D \varphi D \exp \left(\int_{0}^{\frac{1}{T}} d \tau \int d \vec{r} \mathcal{L}_{E}^{p h}+T r \ln L_{E}\right)
\end{aligned}
$$

Here $\mathcal{L}_{E}$ is the Euclidean Lagrangean, $\tau=-i t, T$ is the temperature and

$$
\operatorname{Tr} \hat{O}=2 \operatorname{tr} \int d \vec{r} d \tau<\vec{r} \tau|\hat{O}| \vec{r} \tau>
$$

where $\operatorname{tr}$ denotes trace over the matrix indices, and the multiplier 2 results from the summation over the electronic spins.

To calculate $\operatorname{Tr} \ln L_{E}$, we perform the chiral rotation:

$$
\begin{gathered}
\psi \rightarrow \exp \left(i \frac{\sigma_{3}}{2} \varphi\right) \tilde{\psi}, \\
\bar{\psi} \rightarrow \overline{\tilde{\psi}} \exp \left(i \frac{\sigma_{3}}{2} \varphi\right) .
\end{gathered}
$$

Then:

$$
\exp \left(\operatorname{Tr} \ln L_{E}\right)=J\{\varphi\} \exp \left(\operatorname{Tr} \ln \tilde{L}_{E}\right)
$$


where $J\{\varphi\}$ is the functional Jacobian and the Lagrangean $\tilde{L}_{E}$ is:

$$
\begin{gathered}
\tilde{L}_{E}=\left\{-\sigma_{2}\left[\partial \tau+\frac{V_{F}}{2} \partial_{x} \varphi+e \Phi+t_{y} \operatorname{ch}\left(b_{y}\left(\partial_{y}-i e A_{y}\right)\right)+t_{z} \operatorname{ch}\left(b_{z}\left(\partial_{z}-i e A_{z}\right)\right)\right]-\right. \\
\left.\sigma_{1} V_{F}\left[\partial_{x}-\frac{1}{2 V_{F}} \partial_{\tau} \varphi-i e A_{x}-\frac{t_{y} b_{y}}{2 V_{F}} \partial_{y} \varphi \operatorname{sh}\left(b_{y}\left(\partial_{y}-i e A y\right)\right)-\frac{t_{z} b_{z}}{2 V_{F}} \partial_{z} \varphi \operatorname{sh}\left(b_{z}\left(\partial_{z}-i e A z\right)\right)\right]-\Delta\right\},
\end{gathered}
$$

at $b_{y} \partial_{y} \varphi, b_{z} \partial_{z} \varphi<<1$

We calculate the Jacobian following the Fujikawa scheme [0]. According to this:

$$
J^{-1}\{\varphi\}=\exp \left(-\frac{1}{2} \int d \vec{r} d \tau \varphi \operatorname{Tr} \chi^{*} \sigma_{3} \chi\right)
$$

where $\chi$ stands for the complete set of the asymptotic eigenfunctions of the Lagrangean $\tilde{L_{E}}$ taken in the ultraviolet limit, i.e. at the energies far exceeding $e \phi, e A_{x} V_{F}, t_{y, z}$, and $\Delta$ :

$$
\chi=\exp \left(i\left(k_{x} x+\omega \tau+k_{y} y+k_{z} z\right)\right)
$$

We have:

$$
\begin{gathered}
\operatorname{Tr}\left(\chi^{*} \sigma_{3} \chi\right)=2 \lim _{N \rightarrow \infty} \frac{1}{(2 \pi)^{4}} \int_{-\infty}^{\infty} d k_{x} \int_{-\infty}^{\infty} d \omega \int_{-\pi / b_{y}}^{\pi / b_{y}} d k_{y} \int_{-\pi / b_{z}}^{\pi / b_{z}} d k_{z} \chi^{*} \exp \left(-\frac{\tilde{L_{E}} \sigma_{3} \tilde{L_{E}} \sigma_{3}}{N^{2}}\right) \chi= \\
\frac{2}{b_{y} b_{z}}\left\{-\frac{1}{2 V_{F} \pi} \partial_{\tau}^{2} \varphi-\frac{V_{F}}{2 \pi} \partial_{x}^{2} \varphi-\frac{e}{\pi} E_{x}+\frac{V_{y}^{2}}{2 \pi V_{F}} \partial_{y}^{2} \varphi+\frac{V_{z}^{2}}{2 \pi V_{F}} \partial_{z}^{2} \varphi\right\}- \\
\frac{e b_{y}^{2} t_{y}}{2 V_{F}}<\operatorname{ch}\left(b_{y}\left(\partial_{y}-i e A_{y}\right)\right)>\left(E_{y}-\frac{1}{2 e} \partial_{x y}^{2} \varphi\right) \partial_{y} \varphi-(y \rightarrow z)- \\
i e b_{y} t_{y}<\operatorname{sh}\left(b_{y}\left(\partial_{y}-i e A_{y}\right)\right)>\left(H_{z}+\frac{1}{2 V_{F} e} \partial_{y \tau}^{2} \varphi\right)-(y \rightarrow z)
\end{gathered}
$$

Here

$$
<\hat{f}>=\int d k_{x} d k_{y} d k_{z} d \omega \chi^{*} \hat{f} \chi
$$

and $E_{\nu}, H_{\nu}$ are electric and magnetic fields. It is readily seen, that:

$$
<\operatorname{ch}\left(b_{\nu}\left(\partial_{\nu}-i e A_{\nu}\right)\right)>=<\operatorname{sh}\left(b_{\nu}\left(\partial_{\nu}-i e A_{\nu}\right)\right)>=0
$$

and the Jacobian takes the form:

$$
J\{\varphi\}=\exp \left(-\int d \vec{r} d \tau l\{\varphi\}\right)
$$

where

$$
l\{\varphi\}=\frac{1}{b_{y} b_{z}}\left\{\frac{1}{4 V_{F} \pi}\left(\partial_{\tau} \varphi\right)^{2}+\frac{V_{F}}{4 \pi}\left(\partial_{x} \varphi\right)^{2}-\frac{V_{y}^{2}}{4 \pi V_{F}}\left(\partial_{y} \varphi\right)^{2}-\frac{V_{z}}{4 \pi V_{F}}\left(\partial_{z} \varphi\right)^{2}-\frac{e}{\pi} E_{x} \varphi\right\},
$$

and $V_{y, z}=b_{y, z} t_{y, z}$. 
In a $1 D \mathrm{CDW}\left(V_{y}=V_{z}=0\right)$, the Lagrangean (17) coincides with the one for the chiral anomaly in $1+1$ massless quantum field theory (see e.g. [6]). The connection between the $1 D$ CDW Lagrangean and the chiral anomaly phenomenon was demonstrated in Refs. [7, 8]. The Fröhlich relations (1) evidently follow from the Lagrangean (17).

So, in a Q1D CDW, the chiral anomaly mechanism gives the wrong sign only in the transverse dispersion in Eq. (17). The normal CDW-dispersion is restored due to the phonon terms in the Lagrangean (4) [9]. A modification of Fröhlich relations arises due to the polarization terms in the free energy hidden in $\operatorname{Tr} \ln \tilde{L}_{E}$ (Eq. (9)).

In Ref. [10 the chiral anomaly was studied in a formally similiar system: the Q1D spin density wave. It was claimed in [10] that, under the conditions of a quantum Hall effect, the chiral anomaly produces additional terms linear in $t_{y}$ in Eqs.(1). In this paper, we study the opposite limit of classically "weak" magnetic fields (Eq. (15)) when the terms linear in $t_{y, z}$ turn to zero.

Consider $\operatorname{Tr} \ln \tilde{L}_{E}$ at $|\vec{E}|,|\vec{H}|<<\Delta^{2} / e V_{F}$ and $\vec{\partial} \varphi<<\Delta / V_{F}, \partial_{\tau} \varphi<<\Delta$. The straightforward perturbation expansion (see e.g. [4]) gives:

$$
\begin{gathered}
\operatorname{Tr} \ln \tilde{L}_{E}=\frac{1}{2} \operatorname{Tr} \ln \tilde{L}_{E} \sigma_{3} \tilde{L}_{E} \sigma_{3} \cong \\
\frac{1}{2} \ln \hat{K}_{0}-\frac{1}{2} \int d \vec{r} d \tau\left\{\kappa_{x x} \tilde{E}_{x}^{2}+\kappa_{y y} \tilde{E}_{y}^{2}+\kappa_{z z} \tilde{E}_{z}^{2}+\mu_{z z} \tilde{H}_{z}^{2}+\mu_{y y} \tilde{H}_{y}^{2}\right\}
\end{gathered}
$$

Here

$$
\hat{K}_{0}=-\partial_{\tau}^{2}-V_{F}^{2} \partial_{x}^{2}+\Delta^{2}
$$

and

$$
\begin{gathered}
\kappa_{x x}=\frac{1}{8 \pi^{2}} \frac{\omega_{p}^{2}}{\Delta^{2}}, \quad \kappa_{y y,(z z)}=\kappa_{x x}\left(\frac{t_{y, z}}{\epsilon_{F}}\right)^{2}<<\kappa_{x x}, \\
\mu_{y y} \sim\left(\frac{V_{F}}{c}\right)^{2} \kappa_{z z}, \quad \mu_{z z} \sim\left(\frac{V_{F}}{c}\right)^{2} \kappa_{y y}
\end{gathered}
$$

are the diagonal components of the tensor of the dielectric and magnetic susceptibilities, $\omega_{p}=\left(8 e^{2} V_{F} / \Delta^{2} b_{y} b_{z}\right)^{1 / 2}$ is the plasma frequency, $\epsilon_{F}=V_{F} / b_{x}$ is the Fermi energy, and:

$$
\begin{gathered}
\tilde{E}_{x}=E_{x}-\frac{1}{2 V_{F} e} \partial_{\tau}^{2} \varphi-\frac{V_{F}}{2 e} \partial_{x}^{2} \varphi \\
\tilde{E}_{y}=E_{y}-\frac{V_{F}}{2 e} \partial_{x, y}^{2} \varphi \\
\tilde{E}_{z}=E_{z}-\frac{V_{F}}{2 e} \partial_{x z}^{2} \varphi \\
\tilde{H}_{y}=H_{y}+\frac{i}{2 V_{F} e} \partial_{\tau z}^{2} \varphi \\
\tilde{H}_{z}=H_{z}+\frac{i}{2 V_{F} e} \partial_{\tau y}^{2} \varphi
\end{gathered}
$$


Finally, we substitute Eqs. (18) and (16) into the Eq. (16) and calculating the variation of the free energy functional with respect to the vector and scalar potentials to obtain currents in accordance with Eq.(5). We get in real time:

$$
\begin{gathered}
j_{x}=-\frac{e}{\pi b_{y} b_{z}} \partial_{t} \varphi-\frac{\kappa_{x x} V_{F}}{2 e} \partial_{t}\left(\partial_{x}^{2} \varphi-\frac{1}{V_{F}^{2}} \partial_{t}^{2} \varphi-\frac{V_{y}^{2}}{V_{F}^{2}} \partial_{y}^{2} \varphi-\frac{V_{z}^{2}}{V_{F}^{2}} \partial_{z}^{2} \varphi\right)+\frac{\kappa_{y y}}{2 e} V_{F} \partial_{y y t}^{3} \varphi+\frac{\kappa_{z z}}{2 e} V_{F} \partial_{z z t}^{3} \varphi \\
\rho=\frac{e}{\pi b_{y} b_{z}} \partial_{x} \varphi-\frac{\kappa_{x x} V_{F}}{2 e} \partial_{x}\left(\partial_{x}^{2} \varphi-\frac{1}{V_{F}^{2}} \partial_{t}^{2} \varphi-\frac{V_{y}^{2}}{V_{F}^{2}} \partial_{y}^{2} \varphi-\frac{V_{z}^{2}}{V_{F}^{2}} \partial_{z}^{2} \varphi\right)+\frac{\kappa_{y y}}{2 e} V_{F} \partial_{x y y}^{3} \varphi+\frac{\kappa_{z z}}{2 e} V_{F} \partial_{x z z}^{3} \varphi, \\
j_{y}=-\frac{\kappa_{y y}}{e} V_{F} \partial_{x y t}^{3} \varphi, \\
j_{z}=-\frac{\kappa_{z z}}{e} V_{F} \partial_{x z t}^{3} \varphi .
\end{gathered}
$$

It is evident that Eqs. (22) - (25) automatically satisfy the condition $\partial_{t} \rho+\operatorname{div} \vec{j}=0$.

It is easy to check from the equation of motion:

$$
\frac{\delta}{\delta \varphi}\left\{\int d \vec{r} d t\left[\mathcal{L}^{p h}+l\{\varphi\}\right]+\operatorname{Tr} \ln \tilde{L}\right\}=0
$$

that the polarization correction to Eq. (26), as well as to $j_{x}(22)$ and $\rho(23)$, can be safely neglected in electric fields $|\vec{E}|<<e^{2} / 4 \pi \kappa_{x x} \Delta^{2}$. As actually $\kappa_{x x} \simeq 1$, this inequality always holds in experiment.

Hence, the incommensurate Q1D CDW is described by the equation of motion:

$$
\frac{1}{\alpha^{2}} \partial_{t}^{2} \varphi-\frac{V_{F}^{2}}{2} \partial_{x}^{2} \varphi-\frac{U_{y}^{2}}{2} \partial_{y}^{2} \varphi-\frac{U_{z}^{2}}{2} \partial_{z}^{2} \varphi=\frac{e}{\pi} E_{x}
$$

by the Maxwell equations and by the generalized Fröhlich relations:

$$
\begin{gathered}
j_{x}=-\frac{e}{\pi b_{y} b_{z}} \partial_{t} \varphi-+\frac{\kappa_{y y}}{2 e} V_{F} \partial_{y y t}^{3} \varphi+\frac{\kappa_{z z}}{2 e} V_{F} \partial_{z z t}^{3} \varphi \\
\rho=\frac{e}{\pi b_{y} b_{z}} \partial_{x} \varphi-+\frac{\kappa_{y y}}{2 e} V_{F} \partial_{x y y}^{3} \varphi+\frac{\kappa_{z z}}{2 e} V_{F} \partial_{x z z}^{3} \varphi \\
j_{y}=-\frac{\kappa_{y y}}{e} V_{F} \partial_{x y t}^{3} \varphi \\
j_{z}=-\frac{\kappa_{z z}}{e} V_{F} \partial_{x z t}^{3} \varphi .
\end{gathered}
$$

As an application of our formulas (27), (28), we calculate the Hall constant of the CDW condensate at zero temperature.

Consider the Hall geometry: $H=H_{z}$, the transport CDW current $I_{x}$ flows parallel to the chains in a film with sizes $L_{y}, L_{z}$. As normally $t_{z}<<t_{y}$, we neglect $z$-dependence in Eqs.(27),(28). Perfoming the perturbation expansion over $\kappa_{y y}$ in Maxwell equations, we get:

$$
E_{y}=-2 \pi \kappa_{y y} \frac{V_{F}}{e} \partial_{x y}^{2} \varphi, \quad H_{z}=H_{0}
$$


where $H_{0}$ is the external magnetic field which is assumed to be larger than the magnetic field of the transport current.

In sliding $\operatorname{CDW} \varphi=\varphi\left(x-V_{D} t, y, z\right)$, where $V_{D}\left(E_{x}\right)$ is the nonlinear drift velocity. We get:

$$
E_{y}=\frac{2 \pi \kappa_{y y}}{e^{2}} \frac{V_{F}}{V_{D}} b_{y} b_{z} \frac{\partial j_{x}}{\partial y}
$$

The Hall constant is:

$$
R_{C D W}=\frac{\int_{0}^{L_{y}} d y E_{y}}{c H_{z} I_{x}}=\frac{2 \pi^{2} \kappa_{y y}}{c e^{2} H_{0}} \frac{V_{F}}{V_{D}\left(E_{x}\right)} \frac{b_{y} b_{z}}{L_{z}}
$$

Hence

$$
R_{C D W} I_{x}\left(E_{x}\right)=\text { const } \sim t_{y}^{2} \sim T_{C}^{2}
$$

where $T_{C}$ is the temperature of the Peierls transition. The r.h.s in Eq.(32) is independent of $E_{x}$ which is in a good accordance with the experimental data [3].

Conclusion. We have derived for the first time the generalized Fröhlich relations which relate the transverse currents and fields to the phase gradients in a Q1D CDWconductor. The explanation of the relation between the CDW Hall constant and the nonlinear transport current is found. 


\section{References}

[1] G.Grüner, Density Waves in Solids, Addison-Wasley, Reading, Massachusets,1994.

[2] N.P.Ong, P.Monceau, Solid State Comm., 26, 487 (1978).

[3] G.X.Tessema, N.P.Ong, Phys.Rev. B23, 5607 (1981).

[4] I.V. Krive, I.O. Kulik, A.S. Rozhavsky, Fiz. Nizk. Temp 121123 (1986) (Sov.J. Low Temp. 12, 635, (1986)).

[5] K. Fujikawa, Phys.Rev. D21, 2848 (1980).

[6] R. Roskies, F. Shaposnik, Phys.Rev. D23, 558 (1981).

[7] I.V. Krive, A.S. Rozhavsky, Phys. Lett. A113 313 (1985).

[8] Z.-B. Su, B. Sakita, Phys. Rev. Lett. 56, 780 (1986).

[9] S.N. Artemenko, A.N. Kruglov, Zh. Eksp. Teor. Fiz. 83, 1134 (1983) (Sov. Phys. JETP).

[10] V.M.Yakovenko, H.-S. Goan, cond-mat/9804128, 13 April 1998; Phys.Rev. B58, 10648 (1998). 